\title{
Retrieval conditions and false recognition: Testing the distinctiveness heuristic
}

\author{
DANIEL L. SCHACTER, DANIEL L. CENDAN, CHAD S. DODSON, and ERIN R. CLIFFORD \\ Harvard University, Cambridge, Massachusetts
}

\begin{abstract}
High levels of false recognition are observed after people study lists of semantic associates that all converge on a nonpresented lure word. In previous experiments, we have found that orienting participants to encode distinctive information about study list items by presenting them as pictures as opposed to words produces marked reductions in false recognition. We have suggested that these reductions reflect the operation of a distinctiveness heuristic: Participants demand access to detailed pictorial information in order to support a positive recognition decision. The present experiments provide additional evidence on this point and allow us to distinguish between the distinctiveness heuristic account and an alternative account based on the impoverished encoding of relational information that occurs when one is studying pictures. In Experiment 1, even when only half of the items in a study list were presented as pictures, a general suppression of false recognition was observed that could be attributable to impoverished encoding of relational information. Experiment 2 provided a critical test of the distinctiveness heuristic account: We manipulated test instructions and found that differences in false recognition rates between picture and word encoding were attenuated in a retrieval condition that did not encourage reliance on a distinctiveness heuristic.
\end{abstract}

False recognition occurs when people incorrectly claim that they have previously encountered a novel test item (Underwood, 1965). If participants study large numbers of items that are conceptually or perceptually related to new words on a subsequent recognition test, high levels of false recognition are obtained (e.g., Hintzman, 1988; Koutstaal \& Schacter, 1997; Roediger \& McDermott, 1995; Schacter, Verfaellie, \& Anes, 1997; Shiffrin, Huber, \& Marinelli, 1995). A paradigm used initially by Deese (1959) to induce intrusions on a free recall test, and later adapted by Roediger and McDermott (1995) to examine false alarms on a recognition test, has produced especially striking results (i.e., the Deese/Roediger-McDermott, or DRM, paradigm). After participants studied a series of lists, each containing 15 semantic associates that converged on a nonpresented lure word, rates for false alarm to the critical lure words were comparable to hit rates for studied items, exceeding $80 \%$ in some conditions (cf. Miller \& Wolford, 1999; Roediger \& McDermott, 1999).

It has been suggested that robust false recognition occurs, at least in part, because presentation of numerous strong associates emphasizes semantic features common to all studied words more than it emphasizes distinctive

The second experiment was based on a senior thesis carried out by the second author under the supervision of the first author. Both experiments were supported by National Institute on Aging Grant AG08441 and the Harvard College Research Fund. We thank Anthony Wagner for comments and suggestions. Correspondence concerning this article should be addressed to D. L. Schacter, Department of Psychology,Harvard University, 33 Kirkland St., Cambridge, MA 02138 (e-mail: dls@ wjh.harvard.edu). details of particular items (cf. Brainerd \& Reyna, 1998; Payne, Elie, Blackwell, \& Neuschatz, 1996; Schacter, Norman, \& Koutstaal, 1998; Schacter, Verfaellie, \& Pradere, 1996). To examine this issue, Israel and Schacter (1997) and Schacter, Israel, and Racine (1999) assessed whether false recognition of semantic associates would be reduced when subjects encoded DRM lists along with detailed pictures corresponding to each item. They predicted that presenting items as pictures would increase encoding of distinctive, specific details (Hunt \& McDaniel, 1993), which in turn should make them easier to discriminate from nonstudied semantic associates. A word encoding condition (participants both heard and saw lists of DRM semantic associates) was compared with a picture encoding condition (participants heard the same lists of semantic associates and also saw line drawings corresponding to each word). Following study list presentation, previously studied words, related lures, and unrelated lures were tested on an old/new recognition task, with test items presented as pictures or as words. The results were consistent with the predictions: False recognition of related lures was significantly reduced after pictorial encoding as opposed to word encoding. Dodson and Schacter (2001) reported similar results following a different manipulation of distinctive encoding (saying words aloud as opposed to hearing them; see also Smith \& Hunt, 1998).

We (Dodson \& Schacter, 2001; Israel \& Schacter, 1997; Schacter et al., 1999, Experiment 1) have suggested that reduced false recognition after the encoding of distinctive information depends on a shift in responding that is based on participants' metamemorial assessments of the kinds of information that they feel they should remember. For 
instance, having encountered pictures with each of the presented words, participants in the picture encoding condition might have employed a general rule of thumb whereby they demanded access to detailed pictorial information in order to support a positive recognition decision (see Strack \& Bless, 1994, for discussion of a similar heuristic process, and see Rotello, 1999, for an alternative interpretation of the Strack and Bless experiments). Thus, reduced false recognition might have resulted from a general expectation that a test item should elicit a vivid perceptual recollection if, indeed, it had been presented previously. The participants in standard encoding conditions, by contrast, did not expect to retrieve distinctive representations of previously studied items and, hence, they were much less likely to demand access to detailed recollections. Schacter et al. (1999) referred to the hypothesized "rule of thumb" in the picture encoding condition as a distinctiveness heuristic. When using a distinctiveness heuristic, participants are especially attuned to whether they recollect distinctive details about an item, and they use criteria such as the following: "If I do not remember seeing a picture of an item, it is probably new" (cf. Johnson, Hashtroudi, \& Lindsay, 1993).

Schacter et al. (1999) characterized the distinctiveness heuristic as a retrieval strategy, but there is an alternative account of reduced false recognition following distinctive encoding. Because distinctive encoding manipulations focus participants on the specific features of individual items, they may encode less of the semantic or associative information that relates one word in a list to another (cf. Hunt \& McDaniel, 1993). Thus, rather than reflecting the use of a distinctiveness heuristic during retrieval, reduced false recognition after the studying of pictures may reflect impoverished encoding of semantic or associative relational information.

Data that appear to be inconsistent with this possibility were reported by Schacter et al. (1999, Experiment 2). In Experiment 1 by Schacter et al. (1999; see also Israel \& Schacter, 1997), which provided evidence of reduced false recognition after pictorial encoding, picture versus word encoding was manipulated between subjects. In Experiment 2, picture versus word encoding was manipulated within subjects: Some lists were presented as pictures, others were presented as words, and the two types of lists were intermixed during the study phase. Under these conditions, false recognition of related lure words was not significantly lower after picture encoding than after word encoding. This result would appear to suggest that studying pictures does not result in reduced encoding of semantic or associative information, and it thus undercuts the force of the alternative account suggested above.

However, it is important to consider a further feature of the data from Schacter et al. (1999, Experiment 2). Although false recognition of related lures did not differ between word and picture encoding conditions when some lists were studied as words and others as pictures, the levels of false recognition in both conditions were consider- ably lower than when all the lists were studied as words in Experiment 1 (see Schacter et al., 1999, Figure 1). The presence of some picture lists during the study phase in Experiment 2 might have led to a general reduction of false recognition, perhaps by inducing subjects to focus more on individual items and less on associative or relational information for most or all study lists. Thus, an encodingbased account of false recognition suppression following pictorial encoding in Experiment 1 of Schacter et al. (1999), which had a between-subjects design, remains viable.

Because these suggestions are based on cross-experiment comparisons, they must be treated cautiously. In Experiment 1 , we examined whether including picture lists along with word lists would indeed produce a generalized reduction in false recognition of related lures in comparison with a word encoding condition, as suggested by the cross-experiment comparisons from Schacter et al. (1999).

\section{EXPERIMENT 1}

\section{Method}

Participants. Fifty-four volunteers were recruited from the student population at Harvard University. There were 18 participants in each of the three conditions: word encoding, picture encoding, and $50 \%$ picture encoding. The experiment took approximately $30 \mathrm{~min}$, and each participant was paid $\$ 5$.

Design and Materials. The stimuli consisted of 273 black and white pictures, developed by Israel and Schacter (1997), that were divided into 21 lists of semantically related items. The lists were created using Russell and Jenkins's (1954) word association norms and adapting some of the lists used by Roediger and McDermott (1995). Each list contained 13 items: 12 items were presented at study in order of decreasing associative strength to the nonpresented, related lure item (i.e., the 13th item). For counterbalancing purposes, the 21 lists were divided into three sets of 7 lists so that across participants each list would appear at study and also serve as a source for the new words at test. This rotation also guaranteed that across participants in the $50 \%$ picture condition, the items on each list would be presented as words and pictures at study. The participants studied 14 lists and then completed a recognition test containing items from each of the 21 lists.

During the study phase, the participants saw pictures, saw words, or saw half of the lists as pictures and the remaining lists as words. The lists of related items were presented as a continuous series (i.e., there was no break between the lists). Each study item was presented visually for $1,700 \mathrm{msec}$ and was accompanied with the simultaneous auditory presentation of its name by a male voice. A blank screen with a duration of $1,500 \mathrm{msec}$ separated each study item from the next. During both study and test phases, all items appeared centered on the monitor of an Apple Power Macintosh G3 computer. All words were presented in bold lowercase letters and in 48-point Geneva typefont. The pictures were black and white line drawings and varied in size from $3 \times 3 \mathrm{~cm}$ to $17 \times 18 \mathrm{~cm}$, with a modal size of approximately $10 \times 11 \mathrm{~cm}$.

The recognition test contained 63 words: 28 true targets or studied items (from the 1st and 7th positions of the 14 studied lists); 14 true target controls or unrelated new items (from the 1st and 7th positions of the 7 nonstudied lists); 14 false targets, the critical lures that were semantically related to the items in each of the 14 studied lists; and 7 false target controls, which were the critical lures from each of the 7 nonstudied lists. The test was constructed so that the words were randomly intermixed and also obeyed the following constraints: (1) Each type of item (target, target control, false target, false 
Table 1

Proportion of Items Judged "Old" in the Word, Picture, and $50 \%$ Picture Encoding Conditions

\begin{tabular}{lccc}
\hline & \multicolumn{3}{c}{ Encoding Condition } \\
\cline { 2 - 4 } \multicolumn{1}{c}{ Item Type } & Word & Picture & 50\% Picture \\
\hline False targets & .560 & .266 & .365 \\
False target controls & .175 & .032 & .127 \\
True targets & .671 & .643 & .663 \\
True target controls & .139 & .048 & .063 \\
Corrected false recog. & .385 & .234 & .238 \\
Corrected true recog. & .532 & .595 & .599 \\
\hline
\end{tabular}

Note-False targets are the critical lures from the presented lists; false target controls are the critical lures from the nonpresented lists; true targets are the presented list items; true target controls are unrelated lures.

target control) appeared equally often in each half of the test; (2) no more than two items of the same type appeared consecutively; and (3) items from the same study list were at least eight positions apart.

In sum, the main design consisted of one between-groups variable (encoding condition: word, picture, and $50 \%$ picture) and one within-groups variable (item type).

Procedure. The participants were tested individually and were told to pay attention to the items that they would see because their memories for these items would be tested later. The participants were also informed that they would hear the name of each item. After the study phase, the participants worked on puzzles for $5 \mathrm{~min}$ before receiving the recognition test.

For the recognition test, all of the items were presented as words, and the participants indicated whether each test item was "old" (i.e., it or a picture of it had been seen earlier during the study phase) or "new" (i.e., it or a picture of it had not appeared during the study phase) by pushing the "a" or ";" keys, which had been labeled "old" and "new," respectively. All test words were presented visually in the center of the screen, with the response options "Old" and "New" appearing approximately $2.8 \mathrm{~cm}$ below the word. After each response, the screen cleared and was followed by a 1.5 -sec delay before the presentation of the next test item.

\section{Results}

Table 1 displays the probabilities of responding "old" to the different items in the three different encoding conditions. The central finding is readily apparent in the corrected true and false recognition rates at the bottom of Table 1: Whereas there was little difference across the encoding conditions in corrected true recognition rates, corrected rates for false recognition responses to the related lures were much higher in the word encoding condition than in either the picture or the $50 \%$ picture encoding conditions.

A between-groups analysis of variance (ANOVA) of the uncorrected false recognition rates for the false targets yielded a significanteffect of encoding condition $[F(2,51)=$ $\left.9.61, M S_{\mathrm{e}}=.042, p<.01\right]$. Relative to the false recognition rate in the word encoding condition (.56), participants were significantly less likely to respond "old" to the false targets when they had studied all of the items as pictures $(.27)[F(1,51)=18.56, p<.01]$ or half of the items as pictures $(.37)[F(1,51)=8.14, p<.01]$. There was no significant difference in false recognition rates between the picture and the $50 \%$ picture encoding conditions $[F(1,51)=$ $2.12]$. Because there was some variability across the con- ditions in the rate at which new words were falsely recognized, we also analyzed the corrected false recognition rates for the false targets (i.e., we subtracted the rate for false alarms to the false target controls from the rate for false alarms to the false targets). Although the effect of encoding condition in this ANOVA was marginally significant $\left[F(2,51)=2.78, M S_{\mathrm{e}}=.048, p=.07\right]$, planned comparisons confirmed our predictions: Corrected false recognition rates were significantly lower when participants had studied either all or half of the lists as pictures than when participants had studied all of the lists as words $[F(1,51)=4.28, p<.05$ for the picture vs. word comparison; and $F(1,51)=4.06, p<.05$ for the $50 \%$ picture vs. word comparison].

In contrast to performance for the false targets, as can be seen in Table 1, there was no difference across the encoding conditions in the hit rates for true targets. ANOVAs of both the recognition rates for targets and the corrected true recognition rates (i.e., hit rate for targets minus false alarm rate for target controls) yielded no significant effect of encoding condition [all $F_{\mathrm{s}}(2,51)<1$ ]. Finally, as Schacter et al. (1999) observed within their 50\% picture condition (i.e., their Experiment 2), we also found that false targets that were related to lists of items studied as pictures were falsely recognized at the same rate (.36) as that for false targets related to lists of items studied as words (.37). Similarly, like Schacter et al. (1999), within the 50\% picture condition we found that test items previously studied as pictures were recognized at a higher rate than were test items studied as words $[F(1,17)=4.30, p=.05]$.

\section{Discussion}

Overall, we replicated within a single experiment the trends observed across experiments by Schacter et al. (1999). When participants studied all or $50 \%$ of the list items as pictures, they were overall less likely to falsely recognize related lure items than were participants who studied all of the items as words. As in Schacter et al. (1999, Experiment 2), within the mixed picture-word condition there were no differences in false recognition for lures related to word or picture lists (see Dodson \& Schacter, in press, and Schacter et al., 1999, for further discussion of this finding).

One possible interpretation of the finding that the $50 \%$ picture condition yielded overall lower levels of false recognition than did the word only condition is that the presence of some picture lists resulted in reduced encoding of relational or associative information across all lists. That is, rather than switching from item-based encoding strategies to relational encoding strategies across lists, subjects may have adopted a relatively consistent encoding strategy across lists (we thank John Wixted for suggesting this idea). Thus, the question remains whether suppression of false recognition following study of pictures reflects the use of the retrieval strategy that we have called the distinctivenessheuristic, or whether it results from impoverished encoding of relational/associative information when pictures are studied in some or all lists. 
These considerations highlight that all of our evidence so far for the use of a distinctiveness heuristic comes from studies in which we have manipulated conditions at encoding, such as studying words versus pictures, while holding retrieval conditions constant (Dodson \& Schacter, 2001; Israel \& Schacter, 1997; Schacter et al., 1999). In Experiment 2 , we tested the distinctiveness heuristic hypothesis by varying retrieval conditions as well as encoding conditions. Specifically, we manipulated test instructions to influence the type of information required during retrieval. We compared standard old/new recognition instructions, in which participants must decide whether or not a word has appeared earlier in a study list, with the "meaning recognition" instructions used previously by Brainerd and Reyna (1998). In the meaning recognition condition, participants are instructed to ignore whether an item is old or new and accept all items consistent in meaning with list themes. Thus, the proportion of related lure words endorsed under the meaning recognition instructions provides an index of memory for the semantic theme or gist of study lists that is unopposed by counterveiling influences of item-specific recollection that are (or should be) operative on old/new recognition tests.

In the standard instruction condition, participants need to consider information that is diagnostic of prior study of a specific item, such as whether they can recollect distinctive features associated with the item's presentation, and hence they should invoke a distinctiveness heuristic after picture encoding. In the meaning instruction condition, however, participants need not consider information that is diagnostic of prior study of a particular item and therefore should not invoke a distinctiveness heuristic.

If the reduction of false recognition after pictorial encoding in comparison with word encoding results from the application of a distinctiveness heuristic at retrieval, then analyses of "old" responses to related lure words should reveal an interaction between encoding conditions (word vs. picture) and retrieval conditions (standard vs. meaning). Differences in the proportions of "old" responses to related lures between picture and word encoding under standard test instructions (Israel \& Schacter, 1997; Schacter et al., 1999) should be reduced or eliminated under meaning instructions. Because subjects in the meaning condition no longer need to consider the type of information that is characteristic of recollection of studied items, they should respond by retrieving available semantic gist information following both word and picture encoding.

If, on the other hand, reduced false recognition reflects impoverished encoding of relational/associative information in the picture condition compared with the word condition, parallel results should be observed with standard and meaning instructions. With less semantic information encoded and available after pictorial than after word encoding, participants in the picture encoding condition should be similarly less likely to endorse related lures under both test instructions. Such a finding - parallel effects of the encoding manipulation in both the standard and meaning conditions - would provide compelling evidence against the operation of a distinctiveness heuristic and grounds for rejecting our account. Thus, Experiment 2 provided a strong and critical test of the distinctiveness heuristic hypothesis.

\section{EXPERIMENT 2}

\section{Method}

Participants. Seventy-two Harvard University undergraduates and summer school students participated in this experiment. The participants were recruited from an introductory psychology course and from sign-up sheets posted at Harvard University. The participants who took the introductory psychology course received course credits for their participation. The other participants were paid \$10.

Design and Materials. The basic design was a $2 \times 2$ betweensubjects factorial, with encoding condition (word vs. picture) crossed with retrieval condition (standard instructions vs. meaning instructions). In the picture encoding condition, the participants saw pictures of target items and at the same time heard the corresponding word for each item. In the word encoding condition, the participants saw and heard each word. On the subsequent test, presentation format was the same as during encoding (i.e., in the picture condition, the participants saw a picture and heard the corresponding word; in the word encoding condition, the participants saw and heard each word).

The materials were the same as in Experiment 1, as were the construction of the study lists and tests and the presentation of stimulus materials (except that a female voice presented the stimuli).

Procedure. The study procedure was similar to that of Experiment 1, except that participants worked on brief puzzles (a math subtraction task, a math addition task, and mazes) for 1 min after presentation of each study list. The visual component of each study item was displayed for $1.5 \mathrm{sec}$, with the corresponding auditory component presented simultaneously in a female voice. Approximately $1.5 \mathrm{sec}$ elapsed between these presentations of study items. The presentation of each list took approximately $40 \mathrm{sec}$. Following presentation of all 14 lists, the participants received 3 min to work on the puzzles.

After this filler task, the participants received instructions for either the standard or the meaning recognition test, modified from Brainerd and Reyna (1998). All the participants were informed that they would be presented with a series of items and that some had been presented on the lists that they studied earlier whereas others had not appeared on those lists. Participants who received the standard instructions were asked to indicate whether each item was old or new:

You will be presented with a series of items. Some of these items were presented on the previous lists. Other items were not presented earlier. If you do recognize the item and this think it was OLD (that is, was presented earlier on one of the lists) press the $\mathrm{O}$ key on the computer. When you think that an item is NEW (that is, was not presented earlier on one of the lists) press the $\mathrm{N}$ key on the computer.

For example, suppose you were presented a list like "pine, maple, elm, cottonwood, spruce." If, on the test, you are presented with "pine" you would respond O. However, if presented with "computer" or "oak" you would respond $\mathrm{N}$.

Participants who received the meaning instructions were asked to indicate whether each item was consistent with one of the old themes or concepts from the previously presented lists or was representative of a new theme that did not fit one of the old themes or concepts:

You will be presented with a series of items. Some of these items were presented on the previous lists. Other items were not presented earlier. If you do recognize an item as one of the OLD words that was presented earlier or as a new word that is an example of one of the OLD themes or concepts in the lists presented earlier press the $\mathrm{O}$ key on the computer. When you think that an item is a NEW word that is not an example of 
Table 2

Proportion of Items Judged “Old" as a Function of Encoding and Retrieval Conditions

\begin{tabular}{lccc}
\hline \multirow{2}{*}{ Item Type } & Encoding Condition & \multicolumn{2}{c}{ Retrieval Instructions } \\
\cline { 3 - 4 } False targets & Standard & Meaning \\
\hline \multirow{3}{*}{ False target controls } & Word & .655 & .873 \\
& Word & .278 & .754 \\
True targets & Picture & .175 & .302 \\
& Word & .095 & .254 \\
True target controls & Picture & .774 & .831 \\
& Word & .853 & .905 \\
& Picture & .083 & .266 \\
\hline
\end{tabular}

Note-False targets are the critical lures from the presented lists; false target controls are the critical lures from the nonpresented lists; true targets are the presented list items; true target controls are unrelated lures.

one of the old themes or concepts that you heard before press the $\mathrm{N}$ key on the computer.

For example, suppose you were presented a list like "pine, maple, elm, cottonwood, spruce." If, on the test, you are presented with "pine" or "oak" you would respond O. However, if presented with "computer" you would respond $\mathrm{N}$.

After making each judgment, the participants in both instruction conditions were asked to press the space bar to continue to the next item. All the participants received an example of the task that they were to perform, but none of the items used in the example appeared on the study lists or recognition tests. Furthermore, all participants were told that an old auditory item would never be presented with a new visual item or vice versa.

\section{Results}

Table 2 presents the proportion of "old" responses to false targets, false target controls, true targets, and true target controls as a function of encoding and retrieval conditions.

The critical data concern the proportion of "old" responses to related lures (i.e., false targets) in the four main conditions. As Table 2 indicates, in the standard instruction condition there were many more "old" responses to false targets after word encoding than after picture encoding. By contrast, this large difference was markedly reduced in the meaning instruction condition. A 2 (encoding condition) $\times 2$ (retrieval condition) ANOVA revealed a significant effect of encoding condition $[F(1,68)=33.27$, $\left.M S_{\mathrm{e}}=.033, p<.0001\right]$, indicating more "old" responses to false targets after word encoding than after picture encoding. There was also a main effect of retrieval condition $\left[F(1,68)=65.19, M S_{\mathrm{e}}=.033, p<.005\right]$, indicating more "old" responses with meaning instructions than with standard instructions. Most important, the predicted interaction between encoding and retrieval conditions was reliable $\left[F(1,68)=8.99, M S_{\mathrm{e}}=.033, p<.005\right]$.

A similar pattern emerged when we analyzed corrected recognition scores in which we subtracted the proportion of "old" responses to false target controls from the proportion of "old" responses to false targets. With standard instructions, corrected false recognition was much lower after picture encoding (.183) than after word encoding (.480). But with meaning instructions, there was a much smaller difference between the picture encoding (.500) and word encoding (.571) conditions. A $2 \times 2$ ANOVA revealed main effects of encoding condition $\left[F(1,68)=11.65, M S_{\mathrm{e}}=\right.$ $.053, p<.005]$ and retrieval condition $[F(1,68)=14.29$, $\left.M S_{\mathrm{e}}=.053, p<.0005\right]$, as well as a significant encoding $\times$ retrieval interaction $\left[F(1,68)=4.37, M S_{\mathrm{e}}=.053, p<.05\right]$.

The encoding $\times$ retrieval interaction indicates that, in line with our distinctiveness heuristic account, the reduction of "old" responses to false targets after pictorial encoding in the standard instruction condition was attenuated in the meaning instruction condition. However, in the meaning instruction condition, there was still a trend for fewer "old" responses to false targets after pictorial encoding than after word encoding. Follow-up analyses revealed that this trend achieved borderline significance in the analysis of the uncorrected data $\left[F(1,34)=4.02, M S_{\mathrm{e}}=.032\right.$, $p=.053]$ and did not approach significance in the corrected data $[F(1,34)<1]$.

Analyses of "old" responses to studied items (i.e., true targets) revealed generally parallel effects of the encoding manipulation in the two retrieval conditions. An ANOVA showed a significant effect of encoding condition $[F(1,68)=$ $\left.7.49, M S_{\mathrm{e}}=.014, p<.01\right]$ and a marginal effect of retrieval condition $\left[F(1,68)=3.83, M S_{\mathrm{e}}=.014, p=.054\right]$. The encoding $\times$ retrieval interaction did not approach significance $(F<1)$. Analyses of corrected data in which the proportion of "old" responses to true target controls was subtracted from the proportion of "old" responses to true targets revealed a significant effect of encoding condition $\left[F(1,68)=11.5, M S_{\mathrm{e}}=.038, p<.002\right]$, thus indicating greater discriminability between studied items and new unrelated items after picture encoding than after word encoding. There was a nonsignificanteffect of retrieval condition $[F(1,68)<1.13]$ and a nonsignificant interaction between encoding and retrieval $[F(1,68)<1]$.

\section{GENERAL DISCUSSION}

Replicating previous results (Israel \& Schacter, 1997; Schacter et al., 1999), we found that with standard old/new recognition instructions, the proportion of "old" responses to semantically related lure words was reduced considerably after picture encoding in comparison with word encoding. By contrast, this effect was nearly eliminated in the meaning instruction condition, where participants responded "old" when test probes matched the theme or gist of previously studied items. We predicted this interaction between encoding and retrieval, given our hypothesis that participants rely on a distinctiveness heuristic in the standard instruction condition, but need not and should not invoke the distinctiveness heuristic in the meaning instruction condition. The results thus support the idea that the distinctiveness heuristic is a retrieval strategy in which participants evaluate the presence or absence of expected distinctive information when making an old/new recognition decision. The absence of memory for expected distinctive information is used as evidence that a test item or event is new. 
We noted earlier an alternative account of reduced false recognition after pictorial encoding. Participants in the picture encoding condition, in comparison with those in the word encoding condition, may initially encode less of the associative or relational information that is common to associated items because they focus more on distinctive particulars of each item. Experiment 1 revealed that even when only half of the studied items are pictures, false recognition of related lures is suppressed for both picture and word encoding in comparison with a word encoding condition, raising the possibility that participants tend to focus on item-specific information even when only some of the study lists are presented as pictures.

However, the encoding hypothesis predicts parallel effects of the picture/word encoding manipulation in the two test conditions (i.e., old/new vs. meaning) in Experiment 2, in contrast to the interaction that we observed. Nonetheless, in the meaning instruction condition there were trends for fewer "old" responses to related lures after picture encoding than after word encoding, raising the possibility that some of the effects of picture encoding in the old/new instruction condition are attributable to impoverished encoding of semantic/relational information. Although support for this hypothesis is weak because the results did not attain statistical significance, some contribution of impoverished semantic encoding to reduced false recognition cannot be entirely ruled out, and future studies should examine the issue further. However, the key point from our theoretical perspective is that impoverished semantic encoding cannot account for the interaction between encoding and retrieval conditions that we observed and therefore plays at most a minor role in reducing false recognition after pictorial encoding.

The present experiments thus provide evidence that participants can control the use of the distinctiveness heuristic. Participants invoke the heuristic when retrieval conditions encourage them to consider information that is diagnostic of prior study (old/new instructions), but not when retrieval conditions require them to focus on other kinds of information that are not diagnostic of prior study (meaning instructions).

The results of Experiment 1 are potentially informative in this regard. In light of the results of Experiment 2, it seems plausible that the suppression effects observed in Experiment 1 are attributable, at least in part, to attempts to invoke the distinctiveness heuristic. These findings thus suggest that the distinctive information need not be perfectly diagnostic for participants to use the heuristic: The suppression effect was no different in the picture and $50 \%$ picture conditions. Apparently, after studying some degree of distinctive information, participants heavily weight the presence/absence of memory for this information during the old/new recognition test (see Dodson \& Schacter, in press, for a fuller discussion of diagnosticity and the distinctiveness heuristic).

A number of other investigators have also emphasized that what subjects focus on or look for at retrieval plays an important role in the outcome of decisions about the source of a memory (e.g., Brewer \& Treyens, 1981; Brown, Lewis, \& Monk, 1977; Johnson et al., 1993; Marsh \& Hicks, 1998). An important task for future research is to relate our perspective to these ideas. More generally, our results are consistent with the view that people possess metamemorial knowledge about the aspects of past events that are likely to be remembered (Johnson et al., 1993; Schacter, 2001), but using this knowledge depends on the specific goal of retrieval.

\section{REFERENCES}

Brainerd, C. J., \& Reyna, V. F. (1998). When things that never happened are easier to "remember" than things that did. Psychological Science, 9, 484-489.

Brewer, W. F., \& Treyens, J. C. (1981). Role of schemata in memory for places. Cognitive Psychology, 13, 207-230.

Brown, J., Lewis, V. J., \& Monk, A. F. (1977). Memorability, word frequency and negative recognition. Quarterly Journal of Experimental Psychology, 29, 461-473.

DeEsE, J. (1959). On the prediction of occurrence of particular verbal intrusions in immediate recall. Journal of Experimental Psychology, 58, 17-22.

Dodson, C. S., \& Schacter, D. L. (2001). "If I had said it I would have remembered it": Reducing false memories with a distinctiveness heuristic. Psychological Bulletin \& Review, 8, 155-161.

Dodson, C. S., \& Schacter, D. L. (in press). When false recognition meets metacognition: The distinctiveness heuristic. Journal of Memory \& Language.

Hintzman, D. L. (1988). Judgments of frequency and recognition memory in a multiple-trace memory model. Psychological Review, 95, 528-551.

Hunt, R. R., \& McDaniel, M. A. (1993). The enigma of organization and distinctiveness. Journal of Memory \& Language, 32, 421445 .

ISRAEL, L., \& SChACTER, D. L. (1997). Pictorial encoding reduces false recognition of semantic associates. Psychonomic Bulletin \& Review, 4, 577-581.

Johnson, M. K., Hashtroudi, S., \& Lindsay, D. S. (1993). Source monitoring. Psychological Bulletin, 114, 3-28.

Koutstaal, W., \& Schacter, D. L. (1997). Gist-based false recognition of pictures in older and younger adults. Journal of Memory \& Language, 37, 555-583.

Marsh, R. L., \& Hicks, J. L. (1998). Test formats change sourcemonitoring decision processes. Journal of Experimental Psychology: Learning, Memory, \& Cognition, 24, 1137-1151.

Miller, M. B., \& Wolford, G. L. (1999). The role of criterion shift in false memory. Psychological Review, 106, 398-405.

Payne, D. G., Elie, C. J., Blackwell, J. M., \& Neuschatz, J. S. (1996). Memory illusions: Recalling, recognizing, and recollecting events that never occurred. Journal of Memory \& Language, 35, 261-285.

Roediger, H. L., III, \& McDermott, K. B. (1995). Creating false memories: Remembering words not presented in lists. Journal of Experimental Psychology: Learning, Memory, \& Cognition, 21, 803-814.

Roediger, H. L., III, \& McDermott, K. B. (1999). False alarms about false memories. Psychological Review, 106, 406-410.

Rotello, C. M. (1999). Metacognition and memory for nonoccurrence. Memory, 7, 43-63.

Russell, W. A., \& Jenkins, J. J. (1954). The complete Minnesota norms for responses to 100 words from the Kent-Rosonoff Word Association Test (Tech. Rep. No. 11, Contract N8 ONR 66216). University of Minnesota.

SCHACTER, D. L. (2001). The seven sins of memory: How the mind forgets and remembers. Boston: Houghton Mifflin.

Schacter, D. L., Israel, L., \& Racine, C. (1999). Suppressing false recognition in younger and older adults: The distinctiveness heuristic. Journal of Memory \& Language, 40, 1-24.

Schacter, D. L., Norman, K. A., \& Koutstaal, W. (1998). The cogni- 
tive neuroscience of constructive memory. Annual Review of Psychology, 49, 289-318.

Schacter, D. L., Verfaellie, M., \& Anes, M. D. (1997). Illusory memories in amnesic patients: Conceptual and perceptual false recognition. Neuropsychology, 11, 331-342.

Schacter, D. L., Verfaellie, M., \& Pradere, D. (1996). The neuropsychology of memory illusions: False recall and recognition in amnesic patients. Journal of Memory \& Language, 35, 319-334.

Shiffrin, R., Huber, D. E., \& Marinelli, K. (1995). Effects of category length and strength on familiarity in recognition. Journal of Experimental Psychology: Learning, Memory, \& Cognition, 21, 267-287.
Smith, R. E., \& Hunt, R. R. (1998). Presentation modality affects false memory. Psychonomic Bulletin \& Review, 5, 710-715.

Strack, F., \& Bless, H. (1994). Memory for nonoccurrence: Metacognitive and prepositional strategies. Journal of Memory \& Language, 33, 203-217.

UNDERWOOD, B. J. (1965). False recognition produced by implicit verbal responses. Journal of Experimental Psychology, 70, 122-129.

(Manuscript received August 15, 2000;

revision accepted for publication February 13, 2001.) 